\title{
ADMISSIBLE AND MINIMAX ESTIMATION OF THE PARAMETERS OF THE SELECTED NORMAL POPULATION IN TWO-STAGE ADAPTIVE DESIGNS UNDER REFLECTED NORMAL LOSS FUNCTION
}

BY

HASAN MAZAREI (TEHRAN) AND NADER NEMATOLLAHI (TEHRAN)

\begin{abstract}
In clinical research, one of the key problems is to estimate the effect of the best treatment among the given $k$ treatments in two-stage adaptive design. Suppose the effects of two treatments have normal distributions with means $\theta_{1}$ and $\theta_{2}$, respectively, and common known variance $\sigma^{2}$. In the first stage, random samples of size $n_{1}$ with means $\bar{X}_{1}$ and $\bar{X}_{2}$ are chosen from the two populations. Then the population with the larger (or smaller) sample mean $\bar{X}_{M}$ is selected, and a random sample of size $n_{2}$ with mean $\bar{Y}_{M}$ is chosen from this population in the second stage of design. Our aim is to estimate the mean $\theta_{M}$ (or $\theta_{J}$ ) of the selected population based on $\bar{X}_{M}$ and $\bar{Y}_{M}$ in two-stage adaptive design under the reflected normal loss function. We obtain minimax estimators of $\theta_{M}$ and $\theta_{J}$, and then provide some sufficient conditions for the inadmissibility of estimators of $\theta_{M}$ and $\theta_{J}$. Theoretical results are augmented with a simulation study as well as a real data application.
\end{abstract}

2010 AMS Mathematics Subject Classification: Primary: 62F10, 62F07; Secondary: 62C15, 62C20.

Key words and phrases: Inadmissible estimator, minimax estimator, reflected normal loss function, two-stage adaptive design.

\section{INTRODUCTION}

Estimating the parameters of a selected population in two-stage adaptive design is one of the practical problems in the medical, economics and agriculture fields. In these designs, at first independent samples $X_{i 1}, X_{i 2}, \ldots, X_{i n_{1}}(i=1,2$, $\ldots, k)$ have been drawn from target populations $\Pi_{1}, \Pi_{2}, \ldots, \Pi_{k}$ and then, based on a specific rule, the $M$-th population is selected and a second random sample of size $n_{2}$ is drawn from it. These two samples can be used for estimation of the parameters of the selected population. For example, in the medical research, there are several treatments that scientists are interested in studying them. But, due to various limitations, such as time, the availability of patients, cost, etc., only one or two treatments that have the best response to the observations can be selected 
for further investigation in large-scale medical trials. In general, the best response for continuous and binary cases are defined as the largest mean and the highest proportion, respectively ( $\mathrm{Lu}$ et al. [I]]). In the end, the inference focuses on the selected treatment using both stages' data.

The problem of estimating parameters of a selected population in single-stage design was introduced by Rubinstein [20], [21] and was considered by many researchers. See, for example, Gibbons et al. [6], Vellaisamy [32], [33], Vellaisamy and Punnen [34], Misra et al. [13], [14], Nematollahi and Motamed-Shariati [17], [18], Kumar et al. [8], Arshad and Misra [1]] and Nematollahi [16].

Sarkadi [24] introduced the problem of estimating parameters of the normal population in single-stage design based on the natural selection rule, and following that Dahiya [5], Hsieh [7], Cohen and Sackrowitz [4], Venter [35] and Parsian and Sanjari Farsipour [19] developed some results about estimating parameters of the selected normal population. Misra and Meulen [12] obtained some admissibility results under the reflected normal loss function and Naghizadeh Qomi et al. [15] considered this problem under the reflected normal loss function.

The corresponding two-stage adaptive design, which was introduced by Thall et al. [29], [30], has been discussed extensively by Schaid et al. [25], Sampson and Sill [23], Stallard and Friede [28] and Wu et al. [36]. Lu et al. [11] considered estimating the mean of the selected normal population in two-stage designs under the LINEX loss function and obtained the class of admissible and minimax estimators.

In the literature on the estimation of the parameters of the selected population, most of the considered loss functions are convex and not bounded. However, in some estimation problems, the loss function should be bounded and concave for large values (Tribus and Szonyi [31], León and Wu [10]). For example, in estimating the mean time of treatment $\theta$ of a given illness, the amount of the loss for estimating $\theta$ by an estimator $\delta$ is essentially bounded. Hence, in this paper, we consider the problem of estimating the location parameter of the selected normal population in two-stage adaptive design under the reflected normal loss (RNL) function. The reflected normal loss function was, for the first time, introduced by Spiring [26] and Spiring and Yeung [27]. The general form of this loss function is given by

$$
L(\theta, \delta)=b\left[1-e^{-\Delta^{2} /\left(2 \gamma^{2}\right)}\right],
$$

where $\Delta=\delta-\theta, b$ is the maximum loss and $\gamma$ is a scale parameter. The RNL function is symmetric and bounded and it is useful for the purpose that the amount of loss has a maximum limit. Clearly, the value of $b>0$ does not have any influence on our results, therefore, without loss of generality, we shall take $b=1$ in the rest of the paper.

Let $\Pi_{i}(i=1,2)$ be two normal populations with probability density functions (pdf)

$$
f\left(x \mid \theta_{i}, \sigma^{2}\right)=\frac{1}{\sqrt{2 \pi \sigma^{2}}} e^{-\frac{1}{2 \sigma^{2}}\left(x-\theta_{i}\right)^{2}}, \quad \theta_{i} \in \mathbb{R}, \sigma>0, i=1,2,
$$


where location parameters $\theta_{1}$ and $\theta_{2}$ are unknown and $\sigma^{2}$ is the common known variance. Based on two-stage adaptive design, in the first stage we draw a random sample $X_{i 1}, X_{i 2}, \ldots, X_{i n_{1}}$ of size $n_{1}$ from populations $\Pi_{i}, i=1,2$. For selecting the best population with the larger (or smaller) mean, we use the natural selection rule and select the population corresponding to the larger (or smaller) sample mean. In the second stage, we draw a random sample $Y_{1}, Y_{2}, \ldots, Y_{n_{2}}$ of size $n_{2}$ from the selected population which is independent of the first stage sample. Let $\bar{X}_{1}$ and $\bar{X}_{2}$ be the sample mean of the first stage sample and $\bar{X}_{(1)} \leqslant \bar{X}_{(2)}$ denote the ordered values of $\bar{X}_{1}$ and $\bar{X}_{2}$. For selecting the best population we select the population corresponding to the $\bar{X}_{(2)}\left(\bar{X}_{(1)}\right)$. Therefore, the location parameters corresponding to the larger and smaller selected populations are defined by

$$
\theta_{M}=\left\{\begin{array}{ll}
\theta_{1}, & \bar{X}_{1} \geqslant \bar{X}_{2}, \\
\theta_{2}, & \bar{X}_{1}<\bar{X}_{2},
\end{array} \quad \theta_{J}=\left\{\begin{array}{cc}
\theta_{2}, & \bar{X}_{1} \geqslant \bar{X}_{2}, \\
\theta_{1}, & \bar{X}_{1}<\bar{X}_{2},
\end{array}\right.\right.
$$

respectively. We consider the class of estimators of the parameter $\theta_{M}$ as the convex combination of the sample mean of the selected population $\left(\bar{X}_{M}\right)$ and the sample mean $\left(\bar{Y}_{M}\right)$ of the second stage sample. Therefore, the general form of the estimators is as follows:

$$
\delta_{w}(\mathbf{X}, \mathbf{Y})=w \bar{X}_{M}+(1-w) \bar{Y}_{M}= \begin{cases}w \bar{X}_{1}+(1-w) \bar{Y}_{1}, & \bar{X}_{1} \geqslant \bar{X}_{2}, \\ w \bar{X}_{2}+(1-w) \bar{Y}_{2}, & \bar{X}_{1}<\bar{X}_{2}\end{cases}
$$

where $0 \leqslant w \leqslant 1$. Similar estimators of $\theta_{J}$ can be defined as the convex combination of the sample means of the first and second stages.

In this paper, we want to estimate the parameters $\theta_{M}$ and $\theta_{J}$ of the selected normal population in two-stage adaptive design under the reflected normal loss function. Since the RNL function is bounded, by a result of Basu [2] there exists no uniformly minimum risk unbiased estimator of the unknown parameters. So, we consider minimax and admissible estimation of $\theta_{M}$ and $\theta_{J}$.

To the best of our knowledge, in the literature only Lu et al. [I]] have considered admissibility of linear estimators of the form $\delta_{w}(\mathbf{X}, \mathbf{Y})+c$ of the selected normal population in two-stage adaptive design under the LINEX loss function and showed that $\delta_{w}(\mathbf{X}, \mathbf{Y})$ is not minimax for $\theta_{M}$. In this paper we find minimax estimators of $\theta_{M}$ and $\theta_{J}$ and find sufficient conditions of admissibility of estimators of $\theta_{M}$ and $\theta_{J}$ of the selected normal population in two-stage adaptive design and under the bounded RNL function.

The organization of the paper is as follows. In Section 2, we obtain minimax estimators of $\theta_{M}$ and $\theta_{J}$. In Section B, we employ the technique of Brewster and Zidek [3] to provide some sufficient conditions for the inadmissibility of estimators of location parameters. In Section 4 , we discuss minimaxity and admissibility of estimators of $\theta_{J}$. In Section [5, we compare the risk of the obtained estimators by a simulation study. Also, an application to real data is given in Section 6. Finally, a discussion is given in Section $\square$. 


\section{MINIMAX ESTIMATION}

Suppose random samples of size $n_{1}$ are drawn from two normal populations with pdf ([L.2) in the first stage. Then a random sample of size $n_{2}$ is drawn from the selected population in the second stage of two-stage adaptive design. In this section, we want to show that the convex combination estimator $\delta_{w}(\mathbf{X}, \mathbf{Y})$ given in (ㄴ.4) with $w=\frac{n_{1}}{n_{1}+n_{2}}$ is a minimax estimator of $\theta_{M}$ under the RNL function (ㅁ. $)$. Before obtaining the minimax estimator, we need the following lemma which will be proved in the Appendix.

LEMMA 2.1. Suppose $X_{i 1}, X_{i 2}, \ldots, X_{i n_{1}}, i=1,2$, is a random sample of size $n_{1}$ from the normal population with $p d f(\mathbb{L} .2)$, and $\bar{X}_{(1)} \leqslant \bar{X}_{(2)}$ denote the order statistics of the sample means $\bar{X}_{1}$ and $\bar{X}_{2}$ of these two samples. Select the population corresponding to the largest sample mean $\bar{X}_{M}=\bar{X}_{(2)}$ and draw a random sample $Y_{1}, Y_{2}, \ldots, Y_{n_{2}}$ of size $n_{2}$ from this population, which is independent of the first sample. Let $\bar{Y}_{M}$ be the sample mean of the second stage sample, $\mu=\max \left(\theta_{1}, \theta_{2}\right)-\min \left(\theta_{1}, \theta_{2}\right) \geqslant 0$, and

$$
\sigma_{*}^{2}=\left(\frac{w^{2}}{n_{1}}+\frac{(1-w)^{2}}{n_{2}}\right) \sigma^{2}, \quad \rho=\frac{(1-w)^{2} / n_{2}}{w^{2} / n_{1}+(1-w)^{2} / n_{2}} .
$$

(i) If $S=w \bar{X}_{M}+(1-w) \bar{Y}_{M}-\theta_{M}$, then the pdf of $S$ is given by

$$
f_{S}(s)=\left[\Phi\left(\frac{(1-\rho) s+w \mu}{\sigma_{*} \sqrt{1-\rho^{2}}}\right)+\Phi\left(\frac{(1-\rho) s-w \mu}{\sigma_{*} \sqrt{1-\rho^{2}}}\right)\right] \frac{1}{\sigma_{*}} \varphi\left(\frac{s}{\sigma_{*}}\right)
$$

where $\varphi(\cdot)$ and $\Phi(\cdot)$ denote the normal probability density function and cumulative normal distribution function, respectively.

(ii) Assume that $R\left(\theta_{M}, \delta_{c}\right)$ denotes the risk function of $\delta_{c}=\delta_{w}+c=w \bar{X}_{M}$ $+(1-w) \bar{Y}_{M}+c$ in estimating $\theta_{M}$ under the RNL function (ㅁ. I). Then

$$
R\left(\theta_{M}, \delta_{c}\right)=1-I_{w}\left(c^{2}\right)\{\Phi(d w \mu-e c)+\Phi(-d w \mu-e c)\}
$$

where

$$
\begin{gathered}
d=\frac{1}{\sqrt{1-\rho}} \frac{\sqrt{\gamma^{2}+\sigma_{*}^{2}}}{\sigma_{*} \sqrt{2 \gamma^{2}+\sigma_{*}^{2}(1+\rho)}}, \quad e=\frac{\sigma_{*}^{2}(1-\rho) d}{\gamma^{2}+\sigma_{*}^{2}}, \\
I_{w}\left(c^{2}\right)=\frac{\gamma}{\sqrt{\gamma^{2}+\sigma_{*}^{2}}} e^{-c^{2} /\left(2\left(\gamma^{2}+\sigma_{*}^{2}\right)\right)} .
\end{gathered}
$$

(iii) $R\left(\theta_{M}, \delta_{c}\right)$ is a strictly increasing function of $c$ for $c>0$.

Now, to obtain a minimax estimator of $\theta_{M}$, we use the results of Sackrowitz and Samuel-Cahn [22]. First, we obtain the minimax estimator in the component 
problem for $\theta_{i}, i=1,2$. Let $\bar{X}_{i}, \bar{Y}_{i}$ be the means of the first and second stage samples from the $i$-th population, $i=1,2$. Then $\bar{X}_{i} \sim N\left(\theta_{i}, \frac{\sigma^{2}}{n_{1}}\right)$ and $\bar{Y}_{i} \sim N\left(\theta_{i}, \frac{\sigma^{2}}{n_{2}}\right)$ and $\bar{X}_{i}, \bar{Y}_{i}, i=1,2$, are independent. Assume that $\theta_{i}, i=1,2$, have the following normal prior distributions:

$$
\pi_{i m}\left(\theta_{i}\right)=\frac{1}{\sqrt{2 \pi} m} e^{-\frac{1}{2 m^{2}}\left(\theta_{i}-\eta\right)^{2}}, \quad \eta \in \mathbb{R}, m>0, i=1,2 .
$$

Then the posterior distribution of $\theta_{i}$ given $\left(\bar{X}_{i}, \bar{Y}_{i}\right)=\left(\bar{x}_{i}, \bar{y}_{i}\right)$ is $N\left(\mu^{\prime}, \tau_{m}\right)$ with

$$
\mu^{\prime}=\frac{m^{2}\left(n_{1} \bar{x}_{i}+n_{2} \bar{y}_{i}\right)+\sigma^{2} \eta}{m^{2}\left(n_{1}+n_{2}\right)+\sigma^{2}} \quad \text { and } \quad \tau_{m}=\frac{1}{\left(n_{1}+n_{2}\right) / \sigma^{2}+1 / m^{2}} .
$$

The posterior risk of an estimator $\delta_{i}\left(\bar{X}_{i}, \bar{Y}_{i}\right)$ under the loss function (ILC) is given by the formula

$$
\begin{array}{r}
r\left(\bar{x}_{i}, \bar{y}_{i}, \delta_{i}\left(\bar{x}_{i}, \bar{y}_{i}\right)\right)=1-E\left(\exp \left\{-\frac{1}{2 \gamma^{2}}\left(\delta_{i}\left(\bar{x}_{i}, \bar{y}_{i}\right)-\theta_{i}\right)^{2}\right\} \mid \bar{x}_{i}, \bar{y}_{i}\right) \\
=1-\frac{\gamma}{\sqrt{\gamma^{2}+\tau_{m}}} \exp \left\{-\frac{1}{2\left(\tau_{m}+\gamma^{2}\right)}\left(\delta_{i}\left(\bar{x}_{i}, \bar{y}_{i}\right)-\mu^{\prime}\right)^{2}\right\}
\end{array}
$$

Hence, the posterior risk as a function of $\delta_{i}\left(\bar{x}_{i}, \bar{y}_{i}\right)$ is minimized when $\delta_{i}\left(\bar{x}_{i}, \bar{y}_{i}\right)$ $=E\left(\theta_{i} \mid \bar{x}_{i}, \bar{y}_{i}\right)=\mu^{\prime}$, which is the same as the Bayes estimate of $\theta_{i}$ under the squared error loss (SEL) function. So, the Bayes estimator of $\theta_{i}$ under the RNL function is

$$
\begin{aligned}
\delta_{\pi_{i m}}\left(\bar{X}_{i}, \bar{Y}_{i}\right)=\mu^{\prime} & =E\left(\theta_{i} \mid \bar{X}_{i}, \bar{Y}_{i}\right) \\
& =\frac{m^{2}\left(n_{1} \bar{X}_{i}+n_{2} \bar{Y}_{i}\right)+\sigma^{2} \eta}{m^{2}\left(n_{1}+n_{2}\right)+\sigma^{2}}, \quad i=1,2 .
\end{aligned}
$$

The posterior risk of $\delta_{\pi_{i m}}\left(\bar{X}_{i}, \bar{Y}_{i}\right)$ is given by

$$
r_{\pi_{i m}}\left(\bar{x}_{i}, \bar{y}_{i}, \delta_{\pi_{i m}}\left(\bar{x}_{i}, \bar{y}_{i}\right)\right)=1-\frac{\gamma}{\sqrt{\gamma^{2}+\tau_{m}}} .
$$

Since the posterior risk does not depend on $\left(\bar{x}_{i}, \bar{y}_{i}\right)$, the Bayes risk of $\delta_{\pi_{i m}}\left(\bar{X}_{i}, \bar{Y}_{i}\right)$ is equal to the posterior risk, that is,

$$
r^{*}\left(\pi_{i m}, \delta_{\pi_{i m}}\right)=1-\frac{\gamma}{\sqrt{\gamma^{2}+\tau_{m}}}, \quad i=1,2
$$

Finally, to obtain the Bayes estimator of $\theta_{M}$ under the RNL function and normal prior $\pi_{i_{m}}, i=1,2$, assume that $\theta_{1}$ and $\theta_{2}$ have independent and identical normal 
prior distribution with pdf given in (2.4). Using Lemma 3.2 of [22], we obtain the unique Bayes estimator of $\theta_{M}$ as

$$
\delta_{\pi_{m}}(\mathbf{X}, \mathbf{Y})=\frac{m^{2}\left(n_{1} \bar{X}_{M}+n_{2} \bar{Y}_{M}\right)+\sigma^{2} \eta}{m^{2}\left(n_{1}+n_{2}\right)+\sigma^{2}},
$$

where $\pi_{m}=\left(\pi_{1 m}, \pi_{2 m}\right)$. Note that the posterior risk (2.7) for the component problem is independent of $\left(\bar{x}_{i}, \bar{y}_{i}\right), i=1,2$. Therefore, from Theorem 3.1 of [22] we conclude that the Bayes risk $r^{*}=r^{*}\left(\pi_{m}, \delta_{\pi_{m}}\right)$ of $\delta_{\pi_{m}}(\mathbf{X}, \mathbf{Y})$ is

$$
r^{*}\left(\pi_{m}, \delta_{\pi_{m}}\right)=r^{*}\left(\pi_{i m}, \delta_{\pi_{i m}}\right)=1-\frac{\gamma}{\sqrt{\gamma^{2}+\tau_{m}}}, \quad i=1,2 .
$$

Thus,

$$
\lim _{m \rightarrow \infty} r^{*}\left(\pi_{m}, \delta_{\pi_{m}}\right)=1-\frac{\gamma}{\sqrt{\gamma^{2}+\frac{\sigma^{2}}{n_{1}+n_{2}}}} .
$$

Now, to prove that an estimator $\delta_{m}(\mathbf{X}, \mathbf{Y})$ is a minimax estimator of $\theta_{M}$, by Theorem 3.2 of [22], it suffices to show that for every $\theta=\left(\theta_{1}, \theta_{2}\right)$ we have

$$
R\left(\theta_{M}, \delta_{m}\right) \leqslant \lim _{m \rightarrow \infty} r^{*}\left(\pi_{m}, \delta_{\pi_{m}}\right)=1-\frac{\gamma}{\sqrt{\gamma^{2}+\frac{\sigma^{2}}{n_{1}+n_{2}}}},
$$

where $R\left(\theta_{M}, \delta_{m}\right)$ is the risk function of $\delta_{m}$ under the RNL function.

In the following theorem, according to (2.2) and (2.II), we show that $\delta_{w^{*}}=$ $w^{*} \bar{X}_{M}+\left(1-w^{*}\right) \bar{Y}_{M}$ with $w^{*}=\frac{n_{1}}{n_{1}+n_{2}}$ is a minimax estimator of $\theta_{M}$.

THEOREM 2.1. Under the assumptions of Lemma 2.11, the estimator $\delta_{w^{*}}=$ $w^{*} \bar{X}_{M}+\left(1-w^{*}\right) \bar{Y}_{M}$ with $w^{*}=\frac{n_{1}}{n_{1}+n_{2}}$ is a minimax estimator of $\theta_{M}$ under the RNL function.

Pro of. From equation (2.2) with $c=0$ and $w=w^{*}$ it follows that the risk function $R\left(\theta_{M}, \delta_{w^{*}}\right)$ is of the form

$$
\begin{aligned}
R\left(\theta_{M}, \delta_{w^{*}}\right) & =1-I_{w^{*}}(0)\left\{\Phi\left(d w^{*} \mu\right)+\Phi\left(-d w^{*} \mu\right)\right\} \\
& =1-I_{w^{*}}(0)=1-\frac{\gamma}{\sqrt{\gamma^{2}+\frac{\sigma^{2}}{n_{1}+n_{2}}}}=\lim _{m \rightarrow \infty} r^{*}\left(\pi_{m}, \delta_{\pi_{m}}\right) .
\end{aligned}
$$

Now, by (2.II), the proof is completed .

REMARK 2.1. The risk of $\delta_{w}=w \bar{X}_{M}+(1-w) \bar{Y}_{M}$ for $0 \leqslant w \leqslant 1$ minimizes when $w=w^{*}=\frac{n_{1}}{n_{1}+n_{2}}$. So, the minimax estimator $\delta_{w^{*}}$ of $\theta_{M}$ in two-stage adaptive design dominates the estimators $\delta_{w}$ for $0 \leqslant w \leqslant 1$. Also, by [15], the estimator $\delta_{1}=\bar{X}_{M}=\bar{X}_{(2)}\left(\delta_{w}\right.$ with $\left.w=1\right)$ is a minimax estimator of $\theta_{M}$ in single-stage design. Therefore, the minimax estimator under two-stage adaptive design dominates the minimax estimator of single-stage design. 


\section{SUFFICIENT CONDITIONS FOR INADMISSIBILITY OF ESTIMATORS}

Let $\Pi_{1}$ and $\Pi_{2}$ be two normal populations with associated pdf given by (․2.), and $\bar{X}_{1}, \bar{X}_{2}$ be the first stage sample means of two independent random samples of size $n_{1}$ from these populations. Also, let $\bar{Y}_{M}$ be the mean of the second stage sample of size $n_{2}$ from the selected population. In this section, we want to find

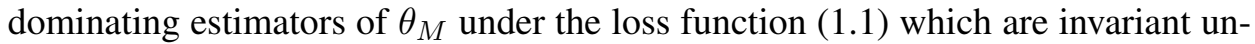
der the location and permutation groups of transformations $\left(\bar{X}_{1}, \bar{X}_{2}\right),\left(\bar{Y}_{1}, \bar{Y}_{2}\right) \rightarrow$ $\left(\bar{X}_{2}+a, \bar{X}_{1}+a\right),\left(\bar{Y}_{2}+a, \bar{Y}_{1}+a\right)$. To this end, we consider the following class of invariant estimators of $\theta_{M}$ :

$$
D_{U}=\left\{\delta_{\psi}=w \bar{X}_{M}+(1-w) \bar{Y}_{M}+\psi(K)\right\},
$$

where $K=\bar{X}_{(1)}-\bar{X}_{(2)}$ and $\psi$ is a real-valued function defined on the interval $(-\infty, 0]$. In this section, by employing the technique of Brewster and Zidek [3], we obtain some sufficient conditions for inadmissibility of invariant estimators of $\theta_{M}$ in the class of estimators $D_{U}$. The following lemma is useful in derivation of a dominating estimator in estimation of $\theta_{M}$.

LEMMA 3.1. Under the assumptions of Lemma 2.1], let $K=\bar{X}_{(1)}-\bar{X}_{(2)}$ and $\psi$ be a real-valued function defined on the interval $(-\infty, 0]$. Also, let

$$
\begin{gathered}
\sigma_{* *}^{2}=\left(\frac{w^{2}}{n_{1}}+\frac{2(1-w)^{2}}{n_{2}}\right) \sigma^{2}, \quad \sigma_{1}^{2}=\frac{\sigma^{2}}{n_{1}}, \\
\psi^{* *}(k)=\frac{\sigma_{*}^{2}+\gamma^{2}}{w \sigma_{1}^{2}} k, \quad \psi^{*}(k)=\frac{\gamma^{2} w k+\sigma_{* *}^{2} \psi(k)}{\sigma_{* *}^{2}+2 \gamma^{2}}
\end{gathered}
$$

and

$$
\eta_{k}(\mu)=\psi^{*}(k)-\frac{\gamma^{2} w \mu}{\sigma_{* *}^{2}+2 \gamma^{2}}\left(1-\frac{2}{1+\exp \left\{2 \mu w \frac{\psi^{* *}(k)-\psi(k)}{\sigma_{* *}^{2}+2 \gamma^{2}}\right\}}\right),
$$

where $\psi^{* *}(k)<k<0$.

(i) For any $k \leqslant 0$, the conditional pdf of $S=w \bar{X}_{M}+(1-w) \bar{Y}_{M}-\theta_{M}$ given $K=k$ is

$$
f_{S \mid K}(s \mid k)=\frac{\sqrt{2}}{\sigma_{* *}} \frac{\varphi\left(\frac{k+\mu}{\sqrt{2} \sigma_{1}}\right) \varphi\left(\frac{\sqrt{2}}{\sigma_{* *}}\left(s+w \frac{k+\mu}{2}\right)\right)+\varphi\left(\frac{k-\mu}{\sqrt{2} \sigma_{1}}\right) \varphi\left(\frac{\sqrt{2}}{\sigma_{* *}}\left(s+w \frac{k-\mu}{2}\right)\right)}{\varphi\left(\frac{k+\mu}{\sqrt{2} \sigma_{1}}\right)+\varphi\left(\frac{k-\mu}{\sqrt{2} \sigma_{1}}\right)} .
$$

(ii) If $\psi(k)>\psi^{* *}(k)$, then $\eta_{k}(\mu)$ is an increasing function of $\mu$ and

$$
\inf _{\mu \geqslant 0} \eta_{k}(\mu)=\eta_{k}(0)=\psi^{*}(k), \quad \sup _{\mu \geqslant 0} \eta_{k}(\mu)=+\infty .
$$


(iii) If $\psi(k) \leqslant \psi^{* *}(k)$, then $\eta_{k}(\mu)$ is a decreasing function of $\mu$ and

$$
\inf _{\mu \geqslant 0} \eta_{k}(\mu)=-\infty, \quad \sup _{\mu \geqslant 0} \eta_{k}(\mu)=\eta_{k}(0)=\psi^{*}(k) .
$$

Pro of. (i) See the Appendix.

(ii) Let $\eta_{k}(\mu)=\psi^{*}(k)-g^{*}(\mu) h_{k}^{*}(\mu)$, where

$$
g^{*}(\mu)=\frac{\gamma^{2} w \mu}{\sigma_{* *}^{2}+2 \gamma^{2}}, \quad h_{k}^{*}(\mu)=1-\frac{2}{1+\exp \left\{2 \mu w \frac{\psi^{* *}(k)-\psi(k)}{\sigma_{* *}^{2}+2 \gamma^{2}}\right\}},
$$

and $l_{k}(\mu)=g^{*}(\mu) h_{k}^{*}(\mu)$. The functions $g^{*}(\mu)$ and $g^{* \prime}(\mu)$ are nonnegative functions of $\mu$ for $\mu \geqslant 0$, and for $\psi(k)>\psi^{* *}(k), h^{*}(\mu)$ and $h^{*^{\prime}}(\mu)$ are nonpositive functions of $\mu$ for $\mu \geqslant 0$. So, we have $l_{k}^{\prime}(\mu)=\left(g^{*}(\mu) h^{*}(\mu)\right)^{\prime}<0$. Therefore, $\eta_{k}(\mu)$ is an increasing function of $\mu$ and hence

$$
\inf _{\mu \geqslant 0} \eta_{k}(\mu)=\eta_{k}(0)=\psi^{*}(k), \quad \sup _{\mu \geqslant 0} \eta_{k}(\mu)=+\infty .
$$

(iii) The proof is similar to the proof of (ii).

In the following theorem, we give sufficient conditions for inadmissibility of invariant estimators $\delta_{\psi}(\mathbf{X}, \mathbf{Y}) \in D_{U}$.

THEOREM 3.1. Suppose $\delta_{\psi}(\mathbf{X}, \mathbf{Y}) \in D_{U}$ is an invariant estimator of $\theta_{M}$. If for every $\theta=\left(\theta_{1}, \theta_{2}\right) \in \mathbb{R}^{2}$

$$
P_{\theta}\left(\psi^{* *}(K)<\psi(K)<\psi^{*}(K)\right)=P_{\theta}\left(\frac{\sigma_{*}^{2}+\gamma^{2}}{w \sigma_{1}^{2}} K<\psi(K)<\frac{w K}{2}\right)>0,
$$

then under the RNL function the estimator $\delta_{\psi}$ is inadmissible for estimating $\theta_{M}$ and is dominated by $\delta_{\psi_{1}}(\mathbf{X}, \mathbf{Y})=w \bar{X}_{M}+(1-w) \bar{Y}_{M}+\psi_{1}(K)$, where

$$
\begin{aligned}
\psi_{1}(K) & = \begin{cases}\psi^{*}(K), & \psi^{* *}(K)<\psi(K)<\psi^{*}(K), \\
\psi(K) & \text { otherwise, }\end{cases} \\
& = \begin{cases}\psi^{*}(K), & \frac{\sigma_{*}^{2}+\gamma^{2}}{w \sigma_{1}^{2}} K<\psi(K)<\frac{w K}{2}, \\
\psi(K) & \text { otherwise }\end{cases}
\end{aligned}
$$

with $\psi^{* *}(K)$ and $\psi^{*}(K)$ defined in (B.2).

P r o of. The difference between risks of $\delta_{\psi}$ and $\delta_{\psi_{1}}$ for $\mu \geqslant 0$ is given by

$$
\begin{aligned}
\Delta(\mu) & =R\left(\theta_{M}, \delta_{\psi}\right)-R\left(\theta_{M}, \delta_{\psi_{1}}\right) \\
& =E\left[1-e^{-\frac{1}{2 \gamma^{2}}(S+\psi(K))^{2}}\right]-E\left[1-e^{-\frac{1}{2 \gamma^{2}}\left(S+\psi_{1}(K)\right)^{2}}\right] \\
& =E\left[e^{-\frac{1}{2 \gamma^{2}}\left(S+\psi_{1}(K)\right)^{2}}-e^{-\frac{1}{2 \gamma^{2}}(S+\psi(K))^{2}}\right]=E\left(D_{\theta}(K)\right) .
\end{aligned}
$$


For every $k \leqslant 0$,

$$
D_{\theta}(k)=E_{\theta}\left[e^{-\frac{1}{2 \gamma^{2}}\left(S+\psi_{1}(k)\right)^{2}}-e^{-\frac{1}{2 \gamma^{2}}(S+\psi(k))^{2}} \mid K=k\right] .
$$

Now, using the inequality

$$
e^{a}-e^{b} \geqslant(a-b) e^{b}, \forall a, b \in \mathbb{R},
$$

we have

$$
\begin{aligned}
D_{\theta}(k) \geqslant & \frac{1}{2 \gamma^{2}} E_{\theta}\left\{\left[(S+\psi(k))^{2}-\left(S+\psi_{1}(k)\right)^{2}\right] e^{-\frac{1}{2 \gamma^{2}}(S+\psi(k))^{2}} \mid K=k\right\} \\
= & \frac{1}{2 \gamma^{2}}\left(\psi(k)-\psi_{1}(k)\right)\left\{\left(\psi(k)+\psi_{1}(k)\right) E_{\theta}\left[e^{-\frac{1}{2 \gamma^{2}}(S+\psi(k))^{2}} \mid K=k\right]\right. \\
& \left.+2 E_{\theta}\left[S e^{-\frac{1}{2 \gamma^{2}}(S+\psi(k))^{2}} \mid K=k\right]\right\} \\
= & \frac{1}{2 \gamma^{2}}\left(\psi(k)-\psi_{1}(k)\right) T(k, \mu)\left\{\psi(k)+\psi_{1}(k)-2 \eta_{k}^{*}(\mu)\right\}
\end{aligned}
$$

where

$$
T(k, \mu)=E_{\theta}\left[e^{-\frac{1}{2 \gamma^{2}}(S+\psi(k))^{2}} \mid K=k\right],
$$

and

$$
\eta_{k}^{*}(\mu)=-\frac{E_{\theta}\left[S e^{-\frac{1}{2 \gamma^{2}}(S+\psi(k))^{2}} \mid K=k\right]}{E_{\theta}\left[e^{-\frac{1}{2 \gamma^{2}}(S+\psi(k))^{2}} \mid K=k\right]} .
$$

By using the conditional pdf of $S \mid K=k$ given in Lemma 3.1 , we find the value of $T(k, \mu)$ as follows:

$$
T(k, \mu)=E_{\theta}\left[e^{-\frac{1}{2 \gamma^{2}}(S+\psi(k))^{2}} \mid K=k\right]=J_{k}^{1}(\mu)+J_{k}^{1}(-\mu),
$$

where

$$
\begin{aligned}
& J_{k}^{1}(\mu)=\frac{\sqrt{2} \gamma}{\sqrt{\sigma_{* *}^{2}+2 \gamma^{2}}} \frac{e^{-\psi^{2}(k) /\left(2 \gamma^{2}\right)}}{1+e^{k \mu / \sigma_{1}^{2}}} \\
& \quad \times \exp \left\{-\frac{w^{2}(k+\mu)^{2}}{4 \sigma_{* *}^{2}}+\frac{\left(\gamma^{2} w(k+\mu)+\sigma_{* *}^{2} \psi(k)\right)^{2}}{2 \sigma_{* *}^{2} \gamma^{2}\left(\sigma_{* *}^{2}+2 \gamma^{2}\right)}\right\} .
\end{aligned}
$$

Similarly, it is easy to verify that

$$
E_{\theta}\left[S e^{-\frac{1}{2 \gamma^{2}}(S+\psi(k))^{2}} \mid K=k\right]=w_{k}^{1}(\mu) J_{k}^{1}(\mu)+w_{k}^{1}(-\mu) J_{k}^{1}(-\mu),
$$


where

$$
w_{k}^{1}(\mu)=-\frac{\sigma_{* *}^{2} \psi(k)+\gamma^{2} w(k+\mu)}{\sigma_{* *}^{2}+2 \gamma^{2}} .
$$

With some simple calculations, we have

$$
\begin{aligned}
\eta_{k}^{*}(\mu)= & \frac{w_{k}^{1}(\mu) J_{k}^{1}(\mu)+w_{k}^{1}(-\mu) J_{k}^{1}(-\mu)}{J_{k}^{1}(\mu)+J_{k}^{1}(-\mu)} \\
= & \frac{\frac{\gamma^{2} w k+\sigma_{* *}^{2} \psi(k)}{\sigma_{* *}^{2}+2 \gamma^{2}}\left(J_{k}^{1}(\mu)+J_{k}^{1}(-\mu)\right)}{J_{k}^{1}(\mu)+J_{k}^{1}(-\mu)} \\
& +\frac{\frac{\gamma^{2} w \mu}{\sigma_{* *}^{2}+2 \gamma^{2}}\left(J_{k}^{1}(\mu)-J_{k}^{1}(-\mu)\right)}{J_{k}^{1}(\mu)+J_{k}^{1}(-\mu)} \\
= & \psi^{*}(k)-\frac{\gamma^{2} w \mu}{\sigma_{* *}^{2}+2 \gamma^{2}}\left(1-\frac{2 J_{k}^{1}(\mu)}{J_{k}^{1}(\mu)+J_{k}^{1}(-\mu)}\right)=\eta_{k}(\mu) .
\end{aligned}
$$

Now, by (B.4) and (B.5), if $\psi(K)>\psi^{*}(K)$ or $\psi(K)<\psi^{* *}(K)$, then $D_{\theta}(K) \geqslant 0$. Also, for $\psi^{* *}(K)<\psi(K)<\psi^{*}(K)$, from (B.5), (B.6) and Lemma B.1] we have

$$
\begin{aligned}
D_{\theta}(k) & \geqslant \frac{1}{2 \gamma^{2}}\left(\psi(k)-\psi^{*}(k)\right) T(k, \mu)\left\{\psi(k)+\psi^{*}(k)-2 \eta_{k}(\mu)\right\} \\
& \geqslant \frac{1}{2 \gamma^{2}}\left(\psi(k)-\psi^{*}(k)\right) T(k, \mu)\left\{\psi(k)-\psi^{*}(k)\right\}>0 .
\end{aligned}
$$

Since $P\left[\psi^{* *}(K)<\psi(K)<\psi^{*}(K)\right]>0$ for every $\theta \in \mathbb{R}^{2}$, we have $\Delta(\mu)>0$, which completes the proof.

REMARK 3.1. Theorem 3.$]$ provides estimators that dominate linear estimators $\delta_{c}(\mathbf{X}, \mathbf{Y})=w \bar{X}_{M}+(1-w) \bar{Y}_{M}+c$ for $c<0$. If we put $\psi(K)=c$ in (B.4), then the dominating estimators are given by

(3.7) $\delta_{c}^{*}(\mathbf{X}, \mathbf{Y})$

$$
=\left\{\begin{array}{l}
\frac{\gamma^{2} w\left(\bar{X}_{(1)}-\bar{X}_{(2)}\right)+\left(\sigma_{* *}^{2}+2 \gamma^{2}\right)\left[w \bar{X}_{M}+(1-w) \bar{Y}_{M}\right]+\sigma_{* *}^{2} c}{\sigma_{* *}^{2}+2 \gamma^{2}}, \\
2 c / w<K<w \sigma_{1}^{2} c /\left(\sigma_{*}^{2}+\gamma^{2}\right), \\
w \bar{X}_{M}+(1-w) \bar{Y}_{M}+c, \quad \text { otherwise. }
\end{array}\right.
$$

Also, since the risk function $R\left(\theta_{M}, \delta_{w}+c\right)$ is an increasing function for $c>0$, part (ii) of Lemma $\mathbf{B}$.$] implies that R\left(\theta_{M}, \delta_{w}+c\right)>R\left(\theta_{M}, \delta_{w}\right)$. So, for $c>0$ the estimator $\delta_{c}(\mathbf{X}, \mathbf{Y})$ is inadmissible and is dominated by $\delta_{w}(\mathbf{X}, \mathbf{Y})=w \bar{X}_{M}+$ $(1-w) \bar{Y}_{M}$. Therefore, the estimator $\delta_{c}(\mathbf{X}, \mathbf{Y})$ for $c \neq 0$ in estimating $\theta_{M}$ under the RNL function is inadmissible. 
REMARK 3.2. Consider the linear combination estimators of the form

$$
\begin{aligned}
\delta_{\psi}(\mathbf{X}, \mathbf{Y}) & =\alpha\left(w \bar{X}_{(1)}+(1-w) \bar{Y}_{M}\right)+(1-\alpha)\left(w \bar{X}_{M}+(1-w) \bar{Y}_{M}\right) \\
& =w \bar{X}_{M}+(1-w) \bar{Y}_{M}+\alpha w\left(\bar{X}_{(1)}-\bar{X}_{M}\right) \\
& =w \bar{X}_{M}+(1-w) \bar{Y}_{M}+\psi(K),
\end{aligned}
$$

where $\frac{1}{2}<\alpha<1$ and

$$
\begin{aligned}
& \psi(K)=\alpha w\left(\bar{X}_{(1)}-\bar{X}_{M}\right)= \begin{cases}\alpha w\left(\bar{X}_{2}-\bar{X}_{1}\right), & \bar{X}_{1}>\bar{X}_{2}, \\
\alpha w\left(\bar{X}_{1}-\bar{X}_{2}\right), & \bar{X}_{1}<\bar{X}_{2},\end{cases} \\
&=\alpha w\left(\bar{X}_{(1)}-\bar{X}_{(2)}\right)=\alpha w K .
\end{aligned}
$$

This estimator is inadmissible for estimating $\theta_{M}$. To obtain an estimator that dominates $\delta_{\psi}$, it suffices to put $\psi(k)=\alpha w K$ in Theorem 3.1 . In this case, the dominating estimator is given by

$$
\begin{aligned}
\delta_{2 \psi}^{*}(\mathbf{X}, \mathbf{Y}) & =w \bar{X}_{M}+(1-w) \bar{Y}_{M}+\psi^{*}(K) \\
& =w \bar{X}_{M}+(1-w) \bar{Y}_{M}+\frac{\alpha \sigma_{* *}^{2}+\gamma^{2}}{\sigma_{* *}^{2}+2 \gamma^{2}} w\left(\bar{X}_{(1)}-\bar{X}_{(2)}\right) .
\end{aligned}
$$

\section{MINIMAX AND INADMISSIBLE ESTIMATORS OF $\theta_{J}$}

In this section, we discuss the minimax and inadmissible estimation of $\theta_{J}$. Using the transformation $\bar{X}_{1} \rightarrow-\bar{X}_{1}$ and $\bar{X}_{2} \rightarrow-\bar{X}_{2}$, and the obtained results of Section $\square$ for $\theta_{M}$, we can easily derive the minimax and inadmissible estimators of $\theta_{J}$. Let $K=\bar{X}_{(1)}-\bar{X}_{(2)}$. Then it can be shown that

$$
R\left(\theta_{J}, U_{J}-\psi(K)\right)=R\left(\theta_{M}, U_{M}+\psi(K)\right),
$$

where $U_{i}=w \bar{X}_{i}+(1-w) \bar{Y}_{i}, i=J, M$, and $\psi$ is a real-valued function defined on $(-\infty, 0]$. So, from the results obtained in Sections $\square$ and $\mathbb{B}$, we have the following results:

(i) Under the reflected normal loss function the estimator $\delta_{w^{*}}^{*}(\mathbf{X}, \mathbf{Y})$ with $w^{*}=\frac{n_{1}}{n_{1}+n_{2}}$ is a minimax estimator of $\theta_{J}$, where

$$
\delta_{w^{*}}^{*}(\mathbf{X}, \mathbf{Y})=w^{*} \bar{X}_{J}+\left(1-w^{*}\right) \bar{Y}_{J}= \begin{cases}w^{*} \bar{X}_{1}+\left(1-w^{*}\right) \bar{Y}_{1}, & \bar{X}_{1}<\bar{X}_{2} \\ w^{*} \bar{X}_{2}+\left(1-w^{*}\right) \bar{Y}_{2}, & \bar{X}_{1} \geqslant \bar{X}_{2}\end{cases}
$$

(ii) Consider the following class of invariant estimators of $\theta_{J}$ :

$$
D_{L}=\left\{\delta_{\varphi}(\overline{\mathbf{X}}, \overline{\mathbf{Y}})=w \bar{X}_{J}+(1-w) \bar{Y}_{J}+\varphi(-K)\right\}
$$


where $\varphi$ is a real-valued function defined on the interval $[0, \infty)$. Let

$\varphi^{*}(-K)=\frac{\sigma_{* *}^{2} \varphi(-K)-\gamma^{2} w K}{\sigma_{* *}^{2}+2 \gamma^{2}} \quad$ and $\quad \varphi^{* *}(-K)=-\frac{\sigma_{*}^{2}+\gamma^{2}}{w \sigma_{1}^{2}} K>-K \geqslant 0$.

Now, if for all $\theta \in \mathbb{R}^{2}$ we have

$$
\begin{aligned}
& P_{\theta}\left(\varphi^{*}(-K)<\varphi(-K)<\varphi^{* *}(-K)\right) \\
&=P_{\theta}\left(-\frac{w K}{2}<\varphi(-K)<-\frac{\sigma_{*}^{2}+\gamma^{2}}{w \sigma_{1}^{2}} K\right)>0,
\end{aligned}
$$

then under the RNL function, the invariant estimator $\delta_{\varphi}$ is inadmissible for estimating $\theta_{J}$ and is dominated by the estimator $\delta_{\varphi_{1}}=w \bar{X}_{J}+(1-w) \bar{Y}_{J}+\varphi_{1}(-K)$, where

$$
\varphi_{1}(-K)= \begin{cases}\varphi^{*}(-K), & -\frac{w K}{2}<\varphi(-K)<-\frac{\sigma_{*}^{2}+\gamma^{2}}{w \sigma_{1}^{2}} K, \\ \varphi(-K), & \text { otherwise. }\end{cases}
$$

(iii) From (ii) we conclude that the estimators $\delta_{2 c}=w \bar{X}_{J}+(1-w) \bar{Y}_{J}+c$ in estimating $\theta_{J}$, for every $c \neq 0$, are inadmissible. Also, the convex linear combination estimators of the form $\delta_{\varphi}(\mathbf{X}, \mathbf{Y})=(1-\alpha) U_{J}+\alpha\left(w \bar{X}_{(2)}+(1-w) Y_{J}\right)=$ $U_{J}-\alpha w K$ for $\frac{1}{2}<\alpha \leqslant 1$ are inadmissible and are dominated by

$$
\delta_{2 \psi}^{*}(\mathbf{X}, \mathbf{Y})=w \bar{X}_{J}+(1-w) Y_{J}-\frac{\alpha \sigma_{* *}^{2}+\gamma^{2}}{\sigma_{* *}^{2}+2 \gamma^{2}} w\left(\bar{X}_{(1)}-\bar{X}_{(2)}\right) .
$$

REMARK 4.1. Following the results of Lehmann [9], under the general loss function $L(\gamma(\theta), \delta(\mathbf{X}))$, an estimator $\delta(\mathbf{X})$ is a risk unbiased estimator of $\gamma(\theta)$ if it satisfies

$$
E_{\theta}[L(\gamma(\theta), \delta(X))] \leqslant E_{\theta}\left[L\left(\gamma^{\prime}(\theta), \delta(X)\right)\right], \forall \gamma(\theta) \neq \gamma^{\prime}(\theta) .
$$

Under the SEL function, the above condition of the risk unbiasedness reduces to the usual condition of unbiasedness, i.e., $E(\delta(\mathbf{X}))=\gamma(\theta)$, and if an estimator $\delta(\mathbf{X})$ is not unbiased, then its bias is given by $\operatorname{Bias}(\delta(\mathbf{X}))=E(\delta(\mathbf{X}))-\gamma(\theta)$. So, the risk unbiased condition and the bias of an estimator depend on the loss function that we use. Under the RNL function, the risk unbiased condition (4.]) reduces to

$$
E\left(\frac{1}{\gamma^{2}}(\delta(\mathbf{X})-\gamma(\theta)) e^{-\frac{1}{2 \gamma^{2}}(\delta(\mathbf{X})-\gamma(\theta))^{2}}\right)=0 .
$$

Therefore, we can define the risk bias of an estimator $\delta(\mathbf{X})$ of $\gamma(\theta)$ under the $R N L$ function as

$$
\operatorname{Risk} \operatorname{Bias}(\delta(\mathbf{X}))=E\left(\frac{1}{\gamma^{2}}(\delta(\mathbf{X})-\gamma(\theta)) e^{-\frac{1}{2 \gamma^{2}}(\delta(\mathbf{X})-\gamma(\theta))^{2}}\right) .
$$


In what follows, we use (4.2) to compute the risk bias of the given estimators in a simulation study.

\section{SIMULATION STUDY}

In this section, we perform a simulation study to compare the single-stage design and two-stage adaptive design estimators under the RNL function. First, we generate random samples of size $N=1000$ from $\Pi_{1} \sim N\left(\theta_{1}, \sigma^{2}\right)$ and $\Pi_{2} \sim$ $N\left(\theta_{2}, \sigma^{2}\right)$ with different values of $\theta_{1}, \theta_{2}$ and $\sigma=1$. These random samples perform our populations. Now, we extract the samples of size $n_{1}=10,20,30$ from both populations and after selecting the target population based on the natural rule, we generate the second stage samples with size $n_{2}=5,10,15,20,25,30,50$, respectively. Then, we compute the estimators $\delta_{1}, \delta_{w^{*}}, \delta_{w^{*}}+c_{i}$ and $\delta_{c_{i}}^{*}$ given by equations (․․) and (B.7) with $w=1, w^{*}=\frac{n_{1}}{n_{1}+n_{2}}$ and $c_{i}=-1,-2$. Also, we obtain the risk functions and risk bias of each of the estimators. To this end, we repeat the simulation study $B=10^{4}$ times and calculate estimated risk function (ERF) and estimated absolute risk bias (EARB). Hence, the simulation study proceeds as follows:

1. For given $\theta_{1}$ and $\theta_{2}$, generate samples of size 1000 from two normal distributions $\Pi_{1} \sim N\left(\theta_{1}, \sigma^{2}\right)$ and $\Pi_{2} \sim N\left(\theta_{2}, \sigma^{2}\right)$ with $\sigma=1$.

2. Use simulation data as two populations, extract first stage design samples of size $n_{1}=10,20,30$ from the two simulated populations.

3. Select the target population based on the natural rule.

4. Extract the second stage design samples from the selected population with sizes $n_{2}=5,10,15,20,25,30,50$, respectively.

5. Calculate the estimators for each sample as follows:

$$
\begin{array}{rlrl}
\delta_{w^{*}}(\mathbf{X}, \mathbf{Y})+c_{i} & =w^{*} \bar{X}_{M}+\left(1-w^{*}\right) \bar{Y}_{M}+c_{i} & \text { with } w^{*} & =\frac{n_{1}}{n_{1}+n_{2}}, \\
c_{i} & =0,-1,-2, \\
\delta_{1}=\bar{X}_{M}, &
\end{array}
$$

$$
=\left\{\begin{array}{l}
\frac{\gamma^{2} w^{*}\left(\bar{X}_{(1)}-\bar{X}_{(2)}\right)+\left(\sigma_{* *}^{2}+2 \gamma^{2}\right)\left[w^{*} \bar{X}_{M}+\left(1-w^{*}\right) \bar{Y}_{M}\right]+\sigma_{* *}^{2} c_{i}}{\sigma_{* *}^{2}+2 \gamma^{2}} \\
2 c_{i} / w^{*}<K<w^{*} \sigma_{1}^{2} c_{i} /\left(\sigma_{*}^{2}+\gamma^{2}\right), \\
w^{*} \bar{X}_{M}+\left(1-w^{*}\right) \bar{Y}_{M}+c_{i}, \quad \text { otherwise },
\end{array}\right.
$$

$$
c_{i}=-1,-2,
$$

where $\delta_{w^{*}}$ and $\delta_{1}$ are minimax estimators for two-stage adaptive design and singlestage design, respectively. Also, $\delta_{c_{i}}^{*}$ dominates the estimator $\delta_{w^{*}}+c_{i}, i=-1,-2$. 
TABLE 1. Comparing the ERF values of six

estimators based on risk criterion for various sample sizes.

\begin{tabular}{|c|c|c|c|c|c|c|c|c|c|}
\hline$\theta_{1}$ & $\theta_{2}$ & $n_{1}$ & $n_{2}$ & $\delta_{w^{*}}$ & $\delta_{1}$ & $\delta_{w^{*}}+c_{1}$ & $\delta_{c_{1}}^{*}$ & $\delta_{w^{*}}+c_{2}$ & $\delta_{c_{2}}^{*}$ \\
\hline 1 & 1 & 10 & 5 & 0.061789 & 0.088678 & 0.535562 & 0.196823 & 0.963455 & 0.531310 \\
\hline 1 & 1 & 10 & 10 & & & & & 916 & \\
\hline 1 & 1 & 10 & 20 & 0.032371 & 0.088678 & 0.585421 & 0.136117 & 0.974042 & 0.370222 \\
\hline 1 & 1 & 20 & 15 & 0.027938 & 0.047421 & 0.577690 & 0.138094 & 0.973812 & 0.391318 \\
\hline 1 & 1 & 20 & 20 & 0.024574 & 0.047421 & 0.585208 & 0.133402 & 0.975057 & 0.364663 \\
\hline 1 & 1 & 20 & 30 & 0.0 & 0.047421 & 30 & 0.108 & 0.976764 & 0.297527 \\
\hline 1 & 1 & 30 & 25 & 0.0 & 0.03 & & 24 & & 0.309773 \\
\hline 1 & 1 & 30 & 30 & 0.016585 & 0.032371 & 0.596 & 0.105392 & 0.977181 & 0.298215 \\
\hline 1 & 1 & 30 & 50 & 0.012 & 0.032371 & 0.606827 & 98 & 724 & 8031 \\
\hline 1 & 1.5 & 10 & 5 & 0.061789 & 0.088678 & 0.574250 & 0.194 & 0.968231 & 0.384570 \\
\hline 1 & 1.5 & 10 & 10 & 0.047421 & 0.088678 & 0.589290 & 0.164323 & 0.972369 & 0.327517 \\
\hline 1 & 1.5 & 10 & 20 & 0.032371 & 0.088678 & 0.605170 & 0.122109 & 0.976235 & 0.253373 \\
\hline 1 & 1.5 & 20 & 15 & 0.0279 & 0.047421 & 0.614660 & 0.093 & 0.977787 & 0.174036 \\
\hline 1 & 1.5 & 20 & 20 & 0.024574 & 0.047421 & 0.617633 & 0.085080 & 8498 & 711 \\
\hline 1 & 1.5 & 20 & 30 & 0.019804 & 0.047421 & 0.621862 & 0.071445 & 0.979471 & 0.131170 \\
\hline 1 & 1.5 & 30 & 25 & 0.018052 & 0.032371 & 0.626703 & 0.060006 & 0.980154 & 0.102239 \\
\hline 1 & 1.5 & 30 & 30 & 0.016585 & 0.032371 & 0.627738 & 0.054803 & 0.980412 & 0.092460 \\
\hline 1 & 1.5 & 30 & 50 & 0.012516 & 0.032371 & 0.630619 & 0.038917 & 0.981110 & 0.064389 \\
\hline 2 & 2 & 10 & 5 & 0.061789 & 0.088678 & 0.535562 & 0.201389 & 0.963455 & 0.529849 \\
\hline 2 & 2 & 10 & 10 & 0.047421 & 0.088678 & 0.560004 & 0.177540 & 0.968916 & 0.460828 \\
\hline 2 & 2 & 10 & 20 & 0.032 & 0.08 & 21 & 0.137408 & 0.974042 & 0.368269 \\
\hline 2 & 2 & 20 & 15 & 0.027938 & 0.047421 & 0.577690 & 0.140998 & 0.973812 & 0.383682 \\
\hline 2 & 2 & 20 & 20 & 0.024574 & 0.047421 & 0.585208 & 0.129592 & 0.975057 & 0.361516 \\
\hline 2 & 2 & 20 & 30 & 0.019804 & 0.047421 & 0.595830 & 0.112403 & 0.976764 & 0.306811 \\
\hline 2 & 2 & 30 & 25 & 0.018052 & 0.032371 & 0.592243 & 0.112654 & 0.976611 & 0.312770 \\
\hline 2 & 2 & 30 & 30 & 0.016585 & 0.032371 & 0.596116 & 0.108950 & 0.977181 & 0.303307 \\
\hline 2 & 2 & 30 & 50 & 0.012516 & 0.032371 & 0.606827 & 0.087081 & 0.978724 & 0.252532 \\
\hline
\end{tabular}

6. Repeat steps $2-5 B=10^{4}$ times and calculate the ERF for the estimator $\delta_{c_{i}}^{*}$ and the EARB for all estimators using the following formulas:

$$
E R F=1-\frac{1}{B} \sum_{j=1}^{B} \exp \left\{-\frac{1}{2 \gamma^{2}}\left(\delta_{c_{i} j}^{*}-\theta_{M j}\right)^{2}\right\}, \quad c_{i}=-1,-2
$$

and

(5.2) $E A R B=\left|\frac{1}{B} \sum_{j=1}^{B} \frac{1}{2 \gamma^{2}}\left(\theta_{M j}-\delta_{i j}(\mathbf{X})\right) \exp \left\{-\frac{1}{2 \gamma^{2}}\left(\delta_{i j}(\mathbf{X})-\theta_{M j}\right)^{2}\right\}\right|$, 
TABLE 2. Comparing the EARB values of six

estimators based on bias criterion for various sample sizes.

\begin{tabular}{|c|c|c|c|c|c|c|c|c|c|}
\hline$\theta_{1}$ & $\theta_{2}$ & $n_{1}$ & $n_{2}$ & $\delta_{w^{*}}$ & $\delta_{1}$ & $\delta_{w^{*}}+c_{1}$ & $\delta_{c_{1}}^{*}$ & $\delta_{w^{*}}+c_{2}$ & $\delta_{c_{2}}^{*}$ \\
\hline 1 & 1 & 10 & 5 & 0.413255 & 0.494090 & 1.433547 & 0.667959 & 1.589200 & \\
\hline 1 & 1 & 10 & 10 & 6 & 0.49 & & & 761 & \\
\hline 1 & 1 & 10 & 20 & 0.295000 & 0.498752 & 1.515 & 0.508211 & 1.561321 & 0.743122 \\
\hline 1 & 1 & 20 & 15 & 0.276628 & 0.354496 & 1.507808 & 0.485661 & 1.573722 & 0.723913 \\
\hline 1 & 1 & 20 & 20 & 0.257343 & 0.354431 & & 393 & 1.568291 & 0.699477 \\
\hline 1 & 1 & 20 & 30 & 0.23 & 0.3559 & & & 434 & 0.625873 \\
\hline 1 & 1 & 30 & 25 & & 0.291 & & & & 1060 \\
\hline 1 & 1 & 30 & 30 & 0.213657 & 0.293332 & 151 & 0.397901 & 1.562465 & 0587147 \\
\hline 1 & 1 & 30 & 50 & 186 & 0.290410 & 017 & & 939 & 0714 \\
\hline 1 & 1.5 & 10 & 5 & 0.419563 & 0.463139 & 2304 & 0.703 & 633 & 0.859409 \\
\hline 1 & 1.5 & 10 & 10 & 0.382777 & 0.456600 & 1.558487 & 0.627024 & 1.494537 & 0.767959 \\
\hline 1 & 1.5 & 10 & 20 & & 0.456814 & & 0.522287 & 1.489110 & 0630165 \\
\hline 1 & 1.5 & 20 & 15 & 0.292322 & 0.346024 & 906 & 0.472 & 1.514996 & 0.561620 \\
\hline 1 & 1.5 & 20 & 20 & 0.272604 & 0.337797 & 1.576621 & 0.43 & 1.513447 & 3214 \\
\hline 1 & 1.5 & 20 & 30 & 0.255212 & 0.338920 & 1.579887 & 0.376633 & 1.514045 & 0.451835 \\
\hline 1 & 1.5 & 30 & 25 & 0.228282 & 0.289210 & & 0.373008 & 1.522808 & 0.429289 \\
\hline 1 & 1.5 & 30 & 30 & 0.220823 & 0.291189 & 1.580 & 0.352887 & 1.522543 & 0.409618 \\
\hline 1 & 1.5 & 30 & 50 & 0.196583 & 0.288408 & 1.587759 & 0.292716 & 1.520354 & 0.337340 \\
\hline 2 & 2 & 10 & 5 & 0.413062 & 0.494795 & 1.435572 & 0.673213 & 1.587199 & 0.988057 \\
\hline 2 & 2 & 10 & 10 & 0.353869 & 0.492938 & 1.475768 & 0.601488 & 1.575913 & 0.892714 \\
\hline 2 & 2 & 10 & 20 & 0.29 & 0.4905 & 89 & 0.510 & 187 & 0.744208 \\
\hline 2 & 2 & 20 & 15 & 0.273427 & 0.356736 & 1.509711 & 0.490984 & 1.572569 & 0.735596 \\
\hline 2 & 2 & 20 & 20 & 0.256350 & 0.353724 & 1.520379 & 0.464389 & 1.567883 & 0.699847 \\
\hline 2 & 2 & 20 & 30 & 0.230922 & 0.355148 & 1.536339 & 0.425382 & 1.560226 & 0.630015 \\
\hline 2 & 2 & 30 & 25 & 0.221259 & 0.289700 & 1.532983 & 0.407758 & 1.565483 & 0.619481 \\
\hline 2 & 2 & 30 & 30 & & 0.291047 & 1.538442 & 0.400312 & 1.562463 & 0.587090 \\
\hline 2 & 2 & 30 & 50 & 0.183952 & 0.291401 & 1.552295 & 0.339430 & 1.555760 & 0.507172 \\
\hline
\end{tabular}

where $\gamma=0.7$ and $\delta_{i j}$ is one of the estimators $\delta_{1}, \delta_{w^{*}}, \delta_{w^{*}}+c_{i}$ and $\delta_{c_{i}}^{*}$ in the $j$-th iteration of sampling. Also, we use equation (2.2) for calculating the risk function of the other estimators.

7. Repeat steps 1-6 for different values of $\theta_{1}=1,2$ and $\theta_{2}=1,1.5,2$.

Tables 1 and $\square$ show the ERF and EARB values of the given estimators. From these tables we observe that the minimax estimator $\delta_{w^{*}}$ is superior to the other estimators in terms of ERF and EARB. Also, for fixed $n_{1}, n_{2}, \theta_{1}$ and $\theta_{2}$, the estimators $\delta_{w^{*}}, \delta_{1}, \delta_{c_{1}}^{*}, \delta_{c_{2}}^{*}, \delta_{w^{*}}+c_{1}$ and $\delta_{w^{*}}+c_{2}$ have increasing order in terms of ERF, respectively. This shows that $\delta_{c_{1}}^{*}\left(\delta_{c_{2}}^{*}\right)$ dominates $\delta_{w^{*}}+c_{1}\left(\delta_{w^{*}}+c_{2}\right)$ (see Remark (3.J). Furthermore, for fixed $n_{1}, n_{2}, \theta_{1}$ and $\theta_{2}$, the estimators $\delta_{w^{*}}, \delta_{1}, \delta_{c_{1}}^{*}$ 
and $\delta_{c_{2}}^{*}$ have increasing order in terms of EARB, respectively, and the EARB values of these estimators are less than those of the estimators $\delta_{w^{*}}+c_{1}$ and $\delta_{w^{*}}+c_{2}$. From Table W we observe that the ERF values of $\delta_{w^{*}}$ and $\delta_{1}$ do not depend on $\mu=\max \left(\theta_{1}, \theta_{2}\right)-\min \left(\theta_{1}, \theta_{2}\right)$ (see (2.2) ), while the ERF values of $\delta_{w^{*}}+c_{1}$ and $\delta_{w^{*}}+c_{2}$ are a nondecreasing function of $\mu$.

To observe the behavior of the ERF and EARB of $\delta_{w^{*}}+c$ with respect to $c$ $(-2<c<0)$, the ERF and EARB of this estimator are plotted in Figure $\mathbb{W}$ for $n_{1}=20, n_{2}=30, \gamma=0.7, \theta_{1}=1$ and $\theta_{2}=1.5$. From this figure we observe that the ERF of $\delta_{w^{*}}+c$ is a decreasing function of $c$ (see Table 四) and the EARB first increases and then decreases.

(a)

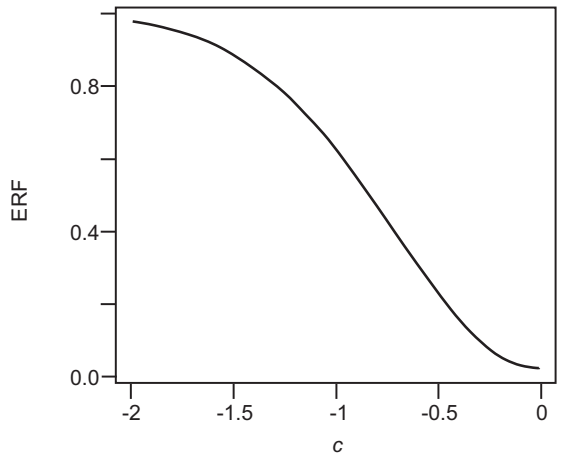

(b)

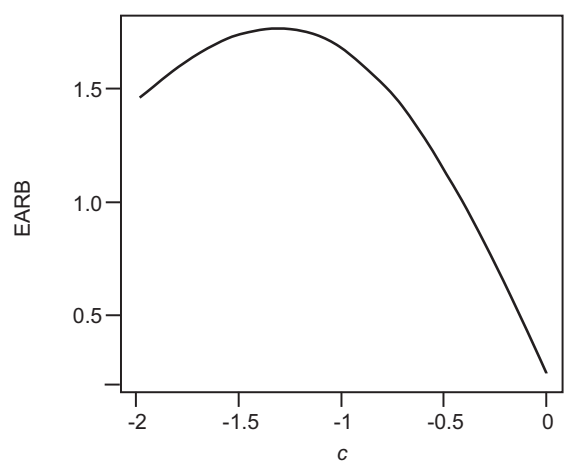

Figure 1. Graph of (a) the ERF, and (b) the EARB of $\delta_{w^{*}}+c$ for $-2<c<0$ and $n_{1}=20, n_{2}=30, \gamma=0.7, \theta_{1}=1, \theta_{2}=1.5$.

\section{APPLICATION}

In this section, we compare the performance of the estimators introduced in Section $\$$ by using a real data. The data is the average weight loss in a finite population consisting of the measurements on the weight loss of 579 participants that were enrolled in a special diet program in a clinical study in Isfahan city of Iran in 2006. This data includes 100 men and 479 women. The weight loss is the difference of the weight of each person before starting the program and after finishing it.

From the Kolmogorov-Smirnov test with a $p$-value of 0.731 for women and 0.980 for men we conclude that the underlying populations of men and women are approximately normal. The assumption of the equality of the population variances is also accepted with $p$-value 0.459 by Levene's test. So, the data can be considered to be a realization of two normal populations $\Pi_{1} \sim N\left(\theta_{1}, \sigma^{2}\right)$ and $\Pi_{2} \sim N\left(\theta_{2}, \sigma^{2}\right)$ with $\left(\hat{\theta}_{1}, \hat{\theta}_{2}\right)=(3.840949,3.736119)$ and pooled sample variance $\widehat{\sigma^{2}}=(2.5)^{2}$.

To estimate the largest average weight loss of two groups (women and men), we extract a sample of size $n_{1}=20$ from each population (group), and then we 
choose a sample of size $n_{2}=30$ from the selected population, i.e., select a population of women when $\overline{x_{1}}>\overline{x_{2}}$ and a population of men when $\overline{x_{1}} \leqslant \overline{x_{2}}$. Then we compute the estimators $\delta_{w^{*}}, \delta_{1}, \delta_{c_{1}}^{*}, \delta_{c_{2}}^{*}, \delta_{w^{*}}+c_{1}$ and $\delta_{w^{*}}+c_{2}$ for $c_{1}=-1$ and $c_{2}=-2$ using (ㄴ.4) and (B.7), respectively.

Table B] summarizes some statistics with the values of the desired estimators based on this sample, where $\theta_{M}=3.840949, \bar{X}_{1}=3.841797, \bar{X}_{2}=3.734493$, $n_{1}=20$ and $n_{2}=30$. This table shows that the two-stage adaptive estimates $\delta_{w^{*}}, \delta_{c_{1}}^{*}$ and $\delta_{c_{2}}^{*}$ are near the true value of the parameter of the selected population, i.e., $\theta_{M}=3.840949$.

TABLE 3. The single-stage and two-stage adaptive estimates of the average weight loss of the selected population.

\begin{tabular}{|cccccc|}
\hline$\delta_{w^{*}}$ & $\delta_{1}$ & $\delta_{w^{*}}+c_{1}$ & $\delta_{c_{1}}^{*}$ & $\delta_{w^{*}}+c_{2}$ & $\delta_{c_{2}}^{*}$ \\
\hline 3.911687 & 4.096379 & 2.911687 & 3.697551 & 1.911687 & 3.482655 \\
\hline
\end{tabular}

To compare the performance of the above estimators, we approximate the risk and bias of the average weight loss of the selected population (women and men). To this end, we perform a simulation study by considering the weight loss data as two populations, and extracting samples of size $n_{1}$ and $n_{2}$ in the first and second stage of sampling, respectively, to compute estimators, risk and risk biases. We repeat this process 10,000 times and calculate the ERF and EARB given in (5.1) and (5.2), respectively, as a comparative tool. To do this, we use the same steps as in Section 5. The results are shown in Tables 4 and 5 where we observe that $\delta_{w^{*}}$ has the smallest ERF and EARB among the six estimators for any sample sizes.

TABLE 4. Comparison of six estimators of the average weight loss of the selected population based on ERF for various sample sizes.

\begin{tabular}{|cccccccc|}
\hline \multicolumn{4}{|c|}{$\theta_{1}=3.7361$} & \multicolumn{3}{c|}{$\theta_{2}=3.8409$} \\
\hline$n_{1}$ & $n_{2}$ & $\delta_{w^{*}}$ & $\delta_{1}$ & $\delta_{w^{*}}+c_{1}$ & $\delta_{c_{1}}^{*}$ & $\delta_{w^{*}}+c_{2}$ & $\delta_{c_{2}}^{*}$ \\
\hline 10 & 5 & 0.061789 & 0.088678 & 0.617866 & 0.251964 & 0.974180 & 0.376701 \\
10 & 10 & 0.047421 & 0.088678 & 0.622622 & 0.222797 & 0.976536 & 0.341151 \\
10 & 20 & 0.032371 & 0.088678 & 0.627799 & 0.176549 & 0.978817 & 0.266394 \\
20 & 15 & 0.027938 & 0.047421 & 0.629360 & 0.149190 & 0.979454 & 0.246953 \\
20 & 20 & 0.024574 & 0.047421 & 0.630555 & 0.142737 & 0.979927 & 0.233309 \\
20 & 30 & 0.019804 & 0.047421 & 0.632266 & 0.121474 & 0.980582 & 0.201216 \\
30 & 25 & 0.018052 & 0.032371 & 0.632898 & 0.114283 & 0.980819 & 0.199039 \\
30 & 30 & 0.016585 & 0.032371 & 0.633430 & 0.106066 & 0.981015 & 0.186342 \\
30 & 50 & 0.012516 & 0.032371 & 0.634913 & 0.085770 & 0.981550 & 0.149221 \\
\hline
\end{tabular}


TABLE 5. Comparison of six estimators of the average weight loss of the selected population based on EARB for various sample sizes.

\begin{tabular}{|cccccccc|}
\hline \multicolumn{4}{|c|}{$\theta_{1}=3.7361$} & \multicolumn{3}{c|}{$\theta_{2}=3.8409$} \\
\hline$n_{1}$ & $n_{2}$ & $\delta_{w^{*}}$ & $\delta_{1}$ & $\delta_{w^{*}}+c_{1}$ & $\delta_{c_{1}}^{*}$ & $\delta_{w^{*}}+c_{2}$ & $\delta_{c_{2}}^{*}$ \\
\hline 10 & 5 & 0.854266 & 0.963364 & 1.191024 & 0.840585 & 1.495613 & 0.934086 \\
10 & 10 & 0.774918 & 0.967146 & 1.254727 & 0.783478 & 1.505901 & 0.878510 \\
10 & 20 & 0.650059 & 0.976764 & 1.353079 & 0.690716 & 1.518354 & 0.761512 \\
20 & 15 & 0.596827 & 0.742356 & 1.357151 & 0.623927 & 1.550353 & 0.703493 \\
20 & 20 & 0.564510 & 0.735805 & 1.394115 & 0.607414 & 1.539330 & 0.680883 \\
20 & 30 & 0.506045 & 0.743844 & 1.439519 & 0.554637 & 1.536949 & 0.624928 \\
30 & 25 & 0.481787 & 0.610765 & 1.439034 & 0.527669 & 1.548947 & 0.599254 \\
30 & 30 & 0.457220 & 0.611865 & 1.457546 & 0.504251 & 1.547747 & 0.573381 \\
30 & 50 & 0.386973 & 0.615708 & 1.502443 & 0.443104 & 1.539037 & 0.498359 \\
\hline
\end{tabular}

\section{DISCUSSION}

In this paper, we have studied the estimation of the parameters $\theta_{M}$ and $\theta_{J}$ in $k=2$ selected normal populations with two-stage adaptive design under the RNL function. In particular, we investigate the minimax estimators of $\theta_{M}$ and $\theta_{J}$ in two-stage adaptive design under the RNL function. We also obtain some sufficient conditions for the inadmissibility of estimators of the location parameters $\theta_{M}$ and $\theta_{J}$. Finally, we compared the performance of our proposed estimators using a simulation study as well as a real data set consisting of the weight loss measurements of two populations in a clinical study in Iran. We showed that the minimax estimator for two-stage adaptive design is better than the other estimators based on estimated risk and estimated risk bias, and also dominates the minimax estimator of the single-stage design.

The results of this paper are obtained for $k=2$ populations. Based on the complexity of obtaining the pdf of $S=w \bar{X}_{M}+(1-w) \bar{Y}_{M}-\theta_{M}$ (i.e., $f_{S}(s)$ ) and the pdf $f_{S \mid K}(s \mid k)$, the extension of the results to the case $k>2$ is very complicated, and further research is needed for this extension.

\section{APPENDIX}

Pro of of Le m ma 2.1. (i) For a proof, we use Lemma 2.1 in Naghizadeh Qomi et al. [15]. Let $\theta_{1}<\theta_{2}$; then $\mu=\theta_{2}-\theta_{1}$. The cumulative distribution function of $S=w \bar{X}_{M}+(1-w) \bar{Y}_{M}-\theta_{M}$ is

$$
\begin{aligned}
F(s)= & P(S \leqslant s)=P\left(w \bar{X}_{M}+(1-w) \bar{Y}_{M}-\theta_{M} \leqslant s\right) \\
= & P\left(\bar{X}_{1}<\bar{X}_{2}, w \bar{X}_{2}+(1-w) \bar{Y}_{2}-\theta_{2} \leqslant s\right) \\
& +P\left(\bar{X}_{1}>\bar{X}_{2}, w \bar{X}_{1}+(1-w) \bar{Y}_{1}-\theta_{1} \leqslant s\right) \\
= & P\left(B_{1}-w \mu<Z_{2} \leqslant s\right)+P\left(B_{2}+w \mu<Z_{1} \leqslant s\right),
\end{aligned}
$$


where $\left(Z_{1}, Z_{2}, B_{1}, B_{2}\right)^{T}$ is defined by

$$
\left[\begin{array}{c}
Z_{1} \\
Z_{2} \\
B_{1} \\
B_{2}
\end{array}\right]=\left[\begin{array}{cccc}
w & 0 & 1-w & 0 \\
0 & w & 0 & 1-w \\
w & 0 & 0 & 1-w \\
0 & w & 1-w & 0
\end{array}\right]\left[\begin{array}{c}
\bar{X}_{1}-\theta_{1} \\
\bar{X}_{2}-\theta_{2} \\
\bar{Y}_{1}-\theta_{1} \\
\bar{Y}_{2}-\theta_{2}
\end{array}\right]
$$

Therefore, $\left(Z_{1}, Z_{2}, B_{1}, B_{2}\right)^{T}$ has the multivariate normal distribution

$$
\left[\begin{array}{l}
Z_{1} \\
Z_{2} \\
B_{1} \\
B_{2}
\end{array}\right] \sim N_{4}\left(\left[\begin{array}{l}
0 \\
0 \\
0 \\
0
\end{array}\right],\left[\begin{array}{cccc}
\sigma_{*}^{2} & 0 & w^{2} \sigma_{1}^{2} & \rho \sigma_{*}^{2} \\
0 & \sigma_{*}^{2} & \rho \sigma_{*}^{2} & w^{2} \sigma_{1}^{2} \\
w^{2} \sigma_{1}^{2} & \rho \sigma_{*}^{2} & \sigma_{*}^{2} & 0 \\
\rho \sigma_{*}^{2} & w^{2} \sigma_{1}^{2} & 0 & \sigma_{*}^{2}
\end{array}\right]\right),
$$

where $\sigma_{1}^{2}=\frac{\sigma^{2}}{n_{1}}$. So,

$$
\begin{aligned}
F(s) & =\int_{-\infty}^{s} \int_{-\infty}^{Z_{2}+w \mu} \frac{1}{2 \pi \sigma_{*}^{2} \sqrt{1-\rho^{2}}} \exp \left\{-\frac{1}{2 \sigma_{*}^{2}\left(1-\rho^{2}\right)}\left(B_{1}^{2}+Z_{2}^{2}-2 \rho Z_{2} B_{1}\right)\right\} d B_{1} d Z_{2} \\
& +\int_{-\infty}^{s} \int_{-\infty}^{Z_{1}-w \mu} \frac{1}{2 \pi \sigma_{*}^{2} \sqrt{1-\rho^{2}}} \exp \left\{-\frac{1}{2 \sigma_{*}^{2}\left(1-\rho^{2}\right)}\left(B_{2}^{2}+Z_{1}^{2}-2 \rho Z_{1} B_{2}\right)\right\} d B_{2} d Z_{1} \\
& =\int_{-\infty}^{s / \sigma_{*}} \varphi(z) \Phi\left(\frac{1-\rho}{\sqrt{1-\rho^{2}}} z+\frac{w \mu}{\sigma_{*} \sqrt{1-\rho^{2}}}\right) d z \\
& +\int_{-\infty}^{s / \sigma_{*}} \varphi(z) \Phi\left(\frac{1-\rho}{\sqrt{1-\rho^{2}}} z-\frac{w \mu}{\sigma_{*} \sqrt{1-\rho^{2}}}\right) d z .
\end{aligned}
$$

Now, if we differentiate both sides with respect to $s$, we get the probability density function of $S$.

(ii) Using the probability density function $f_{S}(s)$ (equation (2.1)), we have

$$
\begin{aligned}
R\left(\theta_{M}, \delta_{c}\right) & =1-E\left[\exp \left\{-\frac{1}{2 \gamma^{2}}\left(w \bar{X}_{M}+(1-w) \bar{Y}_{M}+c-\theta_{M}\right)^{2}\right\}\right] \\
& =1-E\left[\exp \left\{-\frac{1}{2 \gamma^{2}}(s+c)^{2}\right\}\right]=1-\left[I_{1}(c, \mu)+I_{1}(c,-\mu)\right],
\end{aligned}
$$

where

$$
\begin{aligned}
I_{1}(c, \mu)= & \int_{-\infty}^{\infty} \exp \left\{\frac{-(s+c)^{2}}{2 \gamma^{2}}\right\} \Phi\left(\frac{(1-p) s+w \mu}{\sigma_{*} \sqrt{1-p^{2}}}\right) \frac{1}{\sigma_{*}} \varphi\left(\frac{s}{\sigma_{*}}\right) d s \\
= & \frac{1}{\sigma_{*}} \exp \left\{\frac{-c^{2}}{2\left(\gamma^{2}+\sigma_{*}^{2}\right)}\right\} \int_{-\infty}^{\infty} \Phi\left(\frac{(1-\rho) s+w \mu}{\sigma_{*} \sqrt{1-\rho^{2}}}\right) \varphi\left(\frac{\sqrt{\gamma^{2}+\sigma_{*}^{2}}}{\gamma \sigma_{*}}\left(s+\frac{c \sigma_{*}^{2}}{\gamma^{2}+\sigma_{*}^{2}}\right)\right) d s .
\end{aligned}
$$

Let

$$
t=\frac{\sqrt{\gamma^{2}+\sigma_{*}^{2}}}{\gamma \sigma_{*}}\left(s+\frac{c \sigma_{*}^{2}}{\gamma^{2}+\sigma_{*}^{2}}\right) .
$$


Then, using the fact that

$$
\int_{-\infty}^{\infty} \Phi\left(\frac{y+a}{\sqrt{b}}\right) \varphi(y) d y=\Phi\left(\frac{a}{\sqrt{1+b}}\right),
$$

we have

$$
I_{1}(c, \mu)=I_{w}\left(c^{2}\right) \Phi\left(\frac{\frac{w \mu \sqrt{\gamma^{2}+\sigma_{*}^{2}}}{\gamma \sigma_{*}(1-\rho)}-\frac{c \sigma_{*}}{\gamma \sqrt{\gamma^{2}+\sigma_{*}^{2}}}}{\sqrt{\frac{2 \gamma^{2}+\sigma_{*}^{2}(1+\rho)}{\gamma^{2}(1-\rho)}}}\right)=I_{w}\left(c^{2}\right) \Phi(w d \mu-e c) .
$$

So, $R\left(\theta_{M}, \delta_{c}\right)=1-I_{w}\left(c^{2}\right)\{\Phi(w d \mu-e c)+\Phi(-w d \mu-e c)\}$, which completes the proof.

(iii) Since $\Phi(w d \mu-e c)+\Phi(-w d \mu-e c)$ and $I_{w}\left(c^{2}\right)$ are positive and strictly decreasing functions of $c(>0), R\left(\theta_{M}, \delta_{c}\right)$ is also a strictly increasing function of $c(>0)$.

Pro of of Le m maB.1. To prove part (i) of Lemma B.1, first note that the pdf of $K$ is given by (see Lemma 3.1 of [12])

$$
f_{K}(k)=\frac{1}{\sqrt{2} \sigma_{1}}\left[\varphi\left(\frac{k+\mu}{\sqrt{2} \sigma_{1}}\right)+\varphi\left(\frac{k-\mu}{\sqrt{2} \sigma_{1}}\right)\right] .
$$

Now we obtain the conditional cumulative distribution function of $S$ given $K=k$ as follows:

$$
F_{S \mid K}(s \mid k)=P(S \leqslant s \mid K=k)=\frac{1}{f_{K}(k)} \lim _{h \downarrow 0} \frac{N(h)}{h},
$$

where

$$
\begin{aligned}
N(h)= & P(S \leqslant s, k-h<K \leqslant k) \\
= & P\left(w \bar{X}_{2}+(1-w) \bar{Y}_{2}-\theta_{2} \leqslant s, k-h<\bar{X}_{1}-\bar{X}_{2} \leqslant k, \bar{X}_{1}<\bar{X}_{2}\right) \\
& +P\left(w \bar{X}_{1}+(1-w) \bar{Y}_{1}-\theta_{1} \leqslant s, k-h<\bar{X}_{2}-\bar{X}_{1} \leqslant k, \bar{X}_{2}<\bar{X}_{1}\right) \\
= & P\left(Z_{2} \leqslant s, k-h+D_{2}+\mu<D_{1}<k+D_{2}+\mu\right) \\
& +P\left(Z_{1} \leqslant s, k-h+D_{1}-\mu<D_{2}<k+D_{1}-\mu\right) \\
= & \xi_{k}^{1}(\mu)+\xi_{k}^{1}(-\mu),
\end{aligned}
$$

with $D_{i}=\bar{X}_{i}-\theta_{i}$. The joint probability density function of $Z_{2}, D_{1}, D_{2}$ is given by

$$
\left[\begin{array}{l}
Z_{2} \\
D_{1} \\
D_{2}
\end{array}\right] \sim N_{3}\left(\left[\begin{array}{l}
0 \\
0 \\
0
\end{array}\right],\left[\begin{array}{ccc}
\sigma_{*}^{2} & 0 & w \sigma_{1}^{2} \\
0 & \sigma_{1}^{2} & 0 \\
w \sigma_{1}^{2} & 0 & \sigma_{1}^{2}
\end{array}\right]\right) .
$$


Using definitions of $S$ and $K$, we can obtain $\xi_{k}^{1}(\mu)$ as follows:

$$
\begin{aligned}
\xi_{k}^{1}(\mu)= & \int_{-\infty}^{s} \int_{-\infty}^{+\infty} \int_{k-h+D_{2}+\mu}^{k+D_{2}+\mu} f\left(Z_{2} \mid D_{1}, D_{2}\right) f\left(D_{1}\right) f\left(D_{2}\right) d D_{1} d D_{2} d Z_{2} \\
= & \int_{-\infty}^{s} \int_{-\infty}^{+\infty} \int_{k-h+D_{2}+\mu}^{k+D_{2}+\mu} \frac{1}{\sigma_{z}} \varphi\left(\frac{Z_{2}-w D_{2}}{\sigma_{z}}\right) \frac{1}{\sigma_{1}^{2}} \varphi\left(\frac{D_{1}}{\sigma_{1}}\right) \varphi\left(\frac{D_{2}}{\sigma_{1}}\right) d D_{1} d D_{2} d Z_{2} \\
= & \int_{-\infty}^{s} \int_{-\infty}^{+\infty} \frac{1}{\sigma_{z}} \varphi\left(\frac{Z_{2}-w D_{2}}{\sigma_{z}}\right) \frac{1}{\sigma_{1}} \varphi\left(\frac{D_{2}}{\sigma_{1}}\right) \\
& \times\left[\Phi\left(\frac{k+D_{2}+\mu}{\sigma_{1}}\right)-\Phi\left(\frac{k-h+D_{2}+\mu}{\sigma_{1}}\right)\right] d D_{2} d Z_{2},
\end{aligned}
$$

where $\sigma_{z}^{2}=\frac{(1-w)^{2}}{n_{2}} \sigma^{2}$. Now, the limit of $\frac{N(h)}{h}$ can be obtained after some algebraic simplification as follows:

$$
\begin{aligned}
\lim _{h \rightarrow 0} \frac{N(h)}{h}= & \int_{-\infty}^{s} \frac{1}{\sigma_{1} \sigma_{* *}} \varphi\left(\frac{k+\mu}{\sqrt{2} \sigma_{1}}\right) \varphi\left(\frac{\sqrt{2}}{\sigma_{* *}}\left(z_{2}+w \frac{k+\mu}{2}\right)\right) d z_{2} \\
& +\int_{-\infty}^{s} \frac{1}{\sigma_{1} \sigma_{* *}} \varphi\left(\frac{k-\mu}{\sqrt{2} \sigma_{1}}\right) \varphi\left(\frac{\sqrt{2}}{\sigma_{* *}}\left(z_{1}+w \frac{k-\mu}{2}\right)\right) d z_{1} .
\end{aligned}
$$

By differentiating both sides with respect to $s$ and dividing them by the pdf of $f_{K}(k)$, the results will follow.

Acknowledgments. The authors are grateful to the editor and an anonymous referee for making helpful comments and suggestions on an earlier version of this article, which resulted in this improved version.

\section{REFERENCES}

[1] M. Arshad and N. Misra, Estimation after selection from exponential populations with unequal scale parameters, Statist. Papers 57 (3) (2016), pp. 605-621.

[2] D. Basu, A note on the theory of unbiased estimation, Ann. Math. Statist. 26 (1955), pp. 345-348.

[3] J. F. Brewster and J. V. Zidek, Improving on equivariant estimators, Ann. Statist. 2 (1974), pp. 21-38.

[4] A. Cohen and H. B. Sackrowitz, Estimating the mean of the selected population, in: Statistical Decision Theory and Related Topics III, Vol. 1, S. S. Gupta and J. O. Berger (Eds.), Academic Press, New York 1982, pp. 247-270.

[5] R. C. Dahiy a, Estimation of the mean of the selected population, J. Amer. Statist. Assoc. 69 (1974), pp. 226-230.

[6] J. D. Gibbons, I. Olkin, and M. Sobel, Selecting and Ordering Populations: A New Statistical Methodology, Wiley, New York 1977.

[7] H.-K. Hsieh, On estimating the mean of the selected population with unknown variance, Comm. Statist. Theory Methods 10 (18) (1981), pp. 1869-1878. 
[8] S. Kumar, A. K. Mahapatra, and P. Vellaisamy, Reliability estimation of the selected exponential populations, Statist. Probab. Lett. 79 (11) (2009), pp. 1372-1377.

[9] E. L. Lehmann, A general concept of unbiasedness, Ann. Math. Statistics 22 (1951), pp. 587-592.

[10] R. V. León and C. J. Wu, A theory of performance measures in parameter design, Statist. Sinica 2 (2) (1992), pp. 335-358.

[11] X. Lu, A. Sun, and S. S. Wu, On estimating the mean of the selected normal population in two-stage adaptive designs, J. Statist. Plann. Inference 143 (7) (2013), pp. 1215-1220.

[12] N. Misra and E. C. van der Meulen, On estimating the mean of the selected normal population under the LINEX loss function, Metrika 58 (2) (2003), pp. 173-183.

[13] N. Misra, E. C. van der Meulen, and K. Vanden Branden, On estimating the scale parameter of the selected gamma population under the scale invariant squared error loss function, J. Comput. Appl. Math. 186 (1) (2006), pp. 268-282.

[14] N. Misra, E. C. van der Meulen, and K. Vanden Branden, On some inadmissibility results for the scale parameters of selected gamma populations, J. Statist. Plann. Inference 136 (7) (2006), pp. 2340-2351.

[15] M. Naghizadeh Qomi, N. Nematollahi, and A. Parsian, Estimation after selection under reflected normal loss function, Comm. Statist. Theory Methods 41 (6) (2012), pp. 10401051.

[16] N. Nematollahi, Admissible and minimax estimation of the parameter of the selected Pareto population under squared log error loss function, Statist. Papers 58 (2) (2017), pp. 319-339.

[17] N. Nematollahi and F. Motamed-Shariati, Estimation of the scale parameter of the selected gamma population under the entropy loss function, Comm. Statist. Theory Methods 38 (1-2) (2009), pp. 208-221.

[18] N. Nematollahi and F. Motamed-Shariati, Estimation of the parameter of the selected uniform population under the entropy loss function, J. Statist. Plann. Inference 142 (7) (2012), pp. 2190-2202.

[19] A. Parsian and N. Sanjari Farsipour, Estimation of the mean of the selected population under asymmetric loss function, Metrika 50 (2) (1999), pp. 89-107.

[20] D. Rubinstein, Estimation of the reliability of a system in development, Rep. R-61-ELC-1, Tech. Info. Service, General Electric Co., 1961.

[21] D. Rubinstein, Estimation of failure rates in a dynamic reliability program, Rep. 65- RGO-7, Tech. Info. Service, General Electric Co., 1965.

[22] H. B. Sackrowitz and E. Samuel-Cahn, Evaluating the chosen population: A Bayes and minimax approach, in: Adaptive Statistical Procedures and Related Topics, J. van Ryzin (Ed.), IMS Lecture Notes-Monograph Series, Vol. 8, Hayward, CA, 1987, pp. 368-395.

[23] A. R. Sampson and M. W. Sill, Drop-the-losers design: Normal case, Biom. J. 47 (3) (2005), pp. 257-268.

[24] K. Sarkadi, Estimation after selection, Studia Sci. Math. Hungar. 2 (1967), pp. 341-350.

[25] D. J. Schaid, S. Wieand, and T. M. Therneau, Optimal two-stage screening designs for survival comparisons, Biometrika 77 (3) (1990), pp. 507-513.

[26] F. A. Spiring, The reflected normal loss function, Canad. J. Statist. 21 (3) (1993), pp. 321330.

[27] F. A. Spiring and A. S. Yeung, A general class of loss functions with industrial applications, J. Qual. Technol. (30) (1998), pp. 152-162.

[28] N. Stallard and T. Friede, A group-sequential design for clinical trials with treatment selection, Stat. Med. 27 (29) (2008), pp. 6209-6227.

[29] P. F. Thall, R. Simon, and S. S. Ellenberg, Two-stage selection and testing designs for comparative clinical trials, Biometrika 75 (2) (1988), pp. 303-310.

[30] P. F. Thall, R. Simon, and S. S. Ellenberg, A two-stage design for choosing among several experimental treatments and a control in clinical trials, Biometrics 45 (2) (1989), pp. 537-547. 
[31] M. Tribus and G. Szonyi, An alternate view of the Taguchi approach, Quality Progress, May 1989, pp. 46-52.

[32] P. Vellais amy, Inadmissibility results for the selected scale parameters, Ann. Statist. 20 (4) (1992), pp. 2183-2191.

[33] P. Vellaisamy, A note on the estimation of the selected scale parameters, J. Statist. Plann. Inference 55 (1) (1996), pp. 39-46.

[34] P. Vellais amy and A. P. Punnen, Improved estimators for the selected location parameters, Statist. Papers 43 (2) (2002), pp. 291-299.

[35] J. H. Venter, Estimation of the mean of the selected population, Comm. Statist. Theory Methods 17 (3) (1988), pp. 791-805.

[36] S. S. Wu, W. Wang, and M. C. K. Yang, Interval estimation for drop-the-losers designs, Biometrika 97 (2) (2005), pp. 405-418.

Hasan Mazarei

Department of Statistics

Science and Research Branch

Islamic Azad University

Tehran, Iran

E-mail: mazarei_hasan@yahoo.com
Nader Nematollahi Department of Statistics Allameh Tabataba'i University Tehran, Iran E-mail: nematollahi@atu.ac.ir

Received on 15.5.2017;

revised version on 16.5.2018 